Special Issue of the 6th International Congress \& Exhibition (APMAS2016), Maslak, Istanbul, Turkey, June 1-3, 2016

\title{
Vibrating Subsystems of Freight Wagons and Their Beam Models
}

\begin{abstract}
A. BuCHACZ*
Silesian Technical University, Faculty of Mechanical Engineering,

Institute of Engineering Processes Automation and Integrated Manufacturing Systems, 44-100 Gliwice, Poland

The application of analysis of transverse vibrating subsystem of complex microelectromechanical systems by means of the exact and approximate methods were the main purposes of work to solve the task of assignment of frequency-modal analysis and characteristics of mechatronic system. The problems concerned of piezoelectricity and electrostriction and classical and nonclassical methods to solve this problem have been used to obtain the dynamical characteristics in the Gliwice Research Centre. Other diverse problems have been modeled by different methods and next they were examined and analysed. Analysing the diagrams of characteristics of confirmed system, it has been determined that in case of approximate method the resonance frequencies cover with those which have been determined with the exact method. However the values of the characteristic in other areas were different. The main aim of this paper is to compare the transients of characteristics of mechanical subsystem of the transverse vibrating discrete - continuous mechatronic system, obtained by the exact and the approximate Galerkin method and to answer the question - if the method can be used to nominate the characteristics of mechatronic systems. The main subject of deliberation was to determine the flexibility of the mechanical system with constant cross-section using the exact and the Galerkin method. The problems presented in this paper, that means the analysis of subsystem of mechatronic and mechanical complex systems, is however the introduction to the synthesis of transverse vibrating mechatronic systems with assumed frequency spectrum.
\end{abstract}

DOI: 10.12693/APhysPolA.131.62

PACS/topics: 81.05.-t

\section{Analysis of beam as models of subsystems of freight wagons}

In the global case the equation of motion of the beam is considered

$$
E I^{\prime} x x x x(x, t)+\rho F y^{\prime} t t(x, t)=0,
$$

where $y(x, t)$ - deflection at the time moment $t$ of the lining beam section within the distance $x$ from the beginning of the system, $E$ - the Young modulus, $\rho-$ mass density of material of the beam, $I$ - polar inertia moment of the beam cross-section, $F$ - area of the beam cross.

The boundary conditions subsystem of beam system are known and suitably equal in case for:

free end $(F)$

$$
y_{, x x}(x, t)=0, y_{, x x x}(x, t)=0,
$$

clamped end $(C)$ :

$$
y(x, t)=0, y_{, x}(x, t)=0,
$$

pinned end $(P)$ :

$$
y(x, t)=0, y_{, x x}(x, t)=0,
$$

sliding end $(S)$ :

$$
y_{, x}(x, t)=0, y_{, x x x}(x, t)=0 .
$$

The boundary conditions for analyzing beams are combinations of formulae (2)-(5).

\footnotetext{
*e-mail: andrzej.buchacz@polsl.pl
}

The solution of Eq. (1) is the harmonic function in form of

$$
y(x, t)=X(x) \sin \omega t .
$$

Substituting of derivatives of (6) to (1) is obtained

$$
X^{(\mathrm{IV})}(x)-k^{4} X(x)=0 .
$$

The solution $X(x)$ of Eq. (7) is the function of the form

$$
\begin{gathered}
X(x)=A_{1} \sin k x+A_{2} \cos k x \\
\quad+A_{3} \sinh k x+A_{4} \cosh k x .
\end{gathered}
$$

After substitution of (8) into combinations of boundary conditions (2)-(5) for considered beam was received

$$
\boldsymbol{W} \boldsymbol{A}=\mathbf{0},
$$

where $|\boldsymbol{W}|$ - main determinant of set of equation (for example [1-3]), $\boldsymbol{A}=\left[A_{1}, \ldots, A_{4}\right]^{\mathrm{T}}$.

After comparing the characteristic determinant of set (9) to zero

$$
|\boldsymbol{W}|=0,
$$

own values are obtained.

Own functions after relationships between constants $A_{1}, \ldots, A_{4}$ have the form

$$
X_{n}=f\left(\frac{z_{n}}{l} x\right), \quad n=1,2,3, \ldots
$$

The problem of determining of own function were presented for discrete - continuous transverse vibrating mechatronic systems in [1-3].

Taking into consideration that mechanical subsystem of transverse vibrating mechatronic system is extorted with harmonic force in the form $P(t)=P_{0} \sin \omega t$, one boundary condition in (2) or (5) takes the form 


$$
E I y(x, t)^{\prime} x x x x+\rho F y(x, t)^{\prime} t t=P(t) .
$$

The set of equations, after transformations, was obtained in matrix form as

$$
\boldsymbol{W} \boldsymbol{A}=\boldsymbol{F},
$$

where $\boldsymbol{W}, \boldsymbol{A}, \boldsymbol{F}$ - the matrices dependent on one of the boundary conditions of elementary beam.

To qualify constants $A_{1}, \ldots, A_{4}$, we should count combinations of following determinants (e.g. [1-3]):

$$
|\boldsymbol{W}|,\left|\boldsymbol{W}_{1}\right|, \ldots,\left|\boldsymbol{W}_{4}\right| \text {. }
$$

The constants $A_{1}, \ldots, A_{4}$ on the base of combination of (2) $-(5)$ and (for $x=0$ and $x=l$ ), regard as

$$
A_{i}=\frac{\left|\boldsymbol{W}_{i}\right|}{|\boldsymbol{W}|}, i=1, \ldots, 4 .
$$

Substituting expression (14) and (15) to (8) and taking into account (6), deflection beam is

$$
y(x, t)=f(E, I, k, l) P_{0} \sin \omega t .
$$

According to definition of dynamic flexibility, on the basis of (13), it takes form

$$
Y=f(E, I, k, l) \text {. }
$$

Detailed transformations and final sentences of combinations of boundary conditions have been presented in e.g. [1-3].

It has to be considered that if the beam is under the action of moment with continuous factorization along the beam length with the value $F(x) \sin \omega t$ on the length unit, then the equation of motion of the element with length $\mathrm{d} x$ lining in the point $x$ is

$$
E I y_{, x x x x} \mathrm{~d} x+\rho A y_{, t t} \mathrm{~d} x=F(x) \sin \omega t \mathrm{~d} x .
$$

To determine the dynamic flexibility of the factors, which are compatible to concentrate, load $F \sin \omega t$ applied in point $z$ has to be found. The load can be considered as a limit of concentrate load through the length, as follows:

$$
P(x)=\left\{\begin{array}{l}
P / h \text { when } z-h \leq x \leq z, \\
0 \text { in other sections }
\end{array}\right.
$$

and Eq. (18) takes form of

$$
E I y^{\prime} x x x x+\rho A y^{\prime} t t=P_{0} \sin \omega t,
$$
where $P_{0}=\frac{F}{h}$.
Using approximate method - the Galerkin one - the solution of Eq. (20) is given in the form

$$
y(x, t)=\sum_{n=1}^{\infty} y_{n}(x, t)=\sum_{n=1}^{\infty} A^{(n)} f(k, x) \sin \omega t .
$$

Substituting the following derivatives of (21) to (20), the amplitude value $A^{(n)}$ — after transformations - of the vibrations takes form of

$$
A^{(n)}=P_{0} f(a, k, \omega),
$$

where $a=\sqrt{\frac{E I}{\rho F}}$.

Using Eq. (22) and putting it to (21), the dynamical flexibility equals

$$
Y_{x l}^{(n)}=f(a, k, x, l, \omega) .
$$

The absolute value of dynamical flexibility at the end of the beam, i.e. when $x=l$, takes the following form:

$$
\alpha_{Y}^{(l)}=\left|Y_{l l}^{(n)}\right|=f(a, k, l, \omega) .
$$

In general case the dynamical flexibility at the arbitrary point of the beam gets shape of

$$
Y_{x l}=\sum_{n=1}^{\infty} Y_{x l}^{(n)} \text {. }
$$

Proposed problem may be the introduction into solution of reverse task of vibrating subsystems of freight wagons and their beam models.

\section{Acknowledgments}

This work has been conducted as a part of research project PBS2/A6/17/2013 supported by the National Centre for Research and Development in 2013-2016.

\section{References}

[1] A. Buchacz, J. Achiev. Mater. Manufact. Eng. 28, 43 (2008).

[2] A. Buchacz, Proc. Appl. Math. Mech. 9, 373 (2010).

[3] A. Buchacz, Vestnik Krymskoy Akademii NaukSevastopolskoe Otdelene 1, 35 (2010). 